STOCHASTIC MODELING AND CONTROL

BANACH CENTER PUBLICATIONS, VOLUME 122

INSTITUTE OF MATHEMATICS

POLISH ACADEMY OF SCIENCES

WARSZAWA 2020

\title{
FROM SMALL MARKETS TO BIG MARKETS
}

\author{
LAURENCE CARASSUS \\ Léonard de Vinci Pôle Universitaire, Research Center \\ 92 916 Paris La Défense, France \\ and LMR, UMR 9008 Université de Reims-Champagne Ardenne, France \\ ORCID:0000-0002-4428-1735_E-mail: laurence.carassus@devinci.fr
}

\author{
MIKLÓS RÁSONYI \\ Alfréd Rényi Institute of Mathematics \\ Budapest, Hungary \\ ORCID: 0000-0002-3105-4752Ｅ-mail: rasonyi@renyi.hu
}

\begin{abstract}
We study the most famous example of a large financial market: the Arbitrage Pricing Model, where investors can trade in a one-period setting with countably many assets admitting a factor structure. We consider the problem of maximising expected utility in this setting. Besides establishing the existence of optimizers under weaker assumptions than previous papers, we go on studying the relationship between optimal investments in finite market segments and those in the whole market. We show that certain natural (but nontrivial) continuity rules hold: maximal satisfaction, reservation prices and (convex combinations of) optimizers computed in small markets converge to their respective counterparts in the big market.
\end{abstract}

1. Introduction. Arbitrage Pricing Theory (APT) was conceived by [21] in order to derive the conclusions of Capital Asset Pricing Model (see [15, 22]) from alternative assumptions. These remarkable conclusions had a huge bearing on empirical work but they somehow overshadowed the highly inventive model suggested in [21].

Mathematical finance subsequently took up the idea of a market with countably many assets and the theory of large financial markets was founded in [10] and further developed

2010 Mathematics Subject Classification: Primary 93E20, 91B70, 91B16; Secondary 91G10, $46 \mathrm{~B} 09$.

Key words and phrases: Arbitrage Pricing Theory, large markets, maximisation of expected utility.

The paper is in final form and no version of it will be published elsewhere. 
in e.g. [11, 13, 14, 12, 5], just to mention a few. For the sake of generality, continuous trading was assumed in the overwhelming majority of related papers which, again, eclipsed the original setting of 21 .

While the arbitrage theory of the large financial markets has been worked out in [10. 11] satisfactorily in continuous time, other crucial topics — such as utility maximization or superreplication — brought about only dubious conclusions and unsettled questions. Portfolios in finitely many assets were considered in the above references and a natural definition for strategies involving possibly all the assets was missing. Generalized portfolios were introduced (see [7, 5, 16]) as suitable limits of portfolios with finitely many assets. They lacked, however, a clear economic interpretation. In the APT (and, for the moment, only in that model) [20] introduces a straightforward concept of portfolios in infinitely many assets which we will use in the present paper. In [4] it is proved that assuming absence of arbitrage in all of the small markets and under integrability conditions, the no arbitrage condition stated with infinitely many assets also holds true. In the same paper, the authors obtain a dual representation of the superreplication cost of a contingent claim.

In this paper, we investigate the existence of optimizers for utility functions on the whole real line (the positive real axis case was treated in [4]) and we relax some rather stringent conditions imposed in [20, 19. From both a theoretical and a computational viewpoint it is crucial to clarify the relationship between optimal investment in the finite markets and those in the whole market.

In our setup, it is expected that the value functions in finite markets perform asymptotically as well as the value function in the large market. Considering utility indifference prices, these should also converge as the number of assets increases. While these facts are intuitive, no formal justification has been provided so far. We prove these facts in Theorem 3.9 and Corollary 3.11 below. We also prove that certain convex combinations of the optimal portfolios in finite markets perform asymptotically as well as the overall optimizer.

Asymptotic results for superhedging and mean-variance hedging have been obtained in [2, 3. In the utility maximization context the first such result is Theorem 5.3 of [20] where it was shown that there exists a sequence of strategies in finite markets whose values converge to the optimal value. That paper, however, assumed that asset price changes may take arbitrarily large negative and positive values which is a rather strong requirement. Under the more relaxed conditions of the present work we also show the existence of such sequence, moreover, they can be chosen to be averages of finite market optimizers, see Theorem 3.9 below.

Section 2 presents the model and recalls some useful results from [4]. Section 3 contains the main contributions: existence of utility maximization and the asymptotics from small markets to big markets. 
2. The large market model. Let $(\Omega, \mathcal{F}, P)$ be a probability space. We consider a two stage Arbitrage Pricing Model. For any $i \geq 1$, let the return on asset $i$ be given by

$$
\begin{aligned}
& R_{i}=\bar{\beta}_{i}\left(\varepsilon_{i}-b_{i}\right), \quad 1 \leq i \leq m ; \\
& R_{i}=\sum_{j=1}^{m} \beta_{i}^{j}\left(\varepsilon_{j}-b_{j}\right)+\bar{\beta}_{i}\left(\varepsilon_{i}-b_{i}\right), \quad i>m,
\end{aligned}
$$

where the $\left(\varepsilon_{i}\right)_{i \geq 1}$ are random variables and $\left(\bar{\beta}_{i}\right)_{i \geq 1},\left(b_{i}\right)_{i \geq 1},\left(\beta_{i}^{j}\right)_{i>m, 1 \leq j \leq m}$ are constants. We refer to [10, 18, 19] for further discussions on the model.

Assumption 2.1. The $\left(\varepsilon_{i}\right)_{i \geq 1}$ are square-integrable, independent random variables satisfying

$$
E\left(\varepsilon_{i}\right)=0, \quad E\left(\varepsilon_{i}^{2}\right)=1, \quad i \geq 1 .
$$

We consider strategies using potentially infinitely many assets and belonging to

$$
\ell_{2}:=\left\{\left(h_{i}\right)_{i \geq 1} \mid h_{i} \in \mathbb{R}, i \geq 1, \sum_{i=1}^{\infty} h_{i}^{2}<\infty\right\},
$$

which is an Hilbert space with the norm $\|h\|_{\ell_{2}}:=\sqrt{\sum_{i=1}^{\infty} h_{i}^{2}}$.

Let $L^{2}(\Omega, \mathcal{F}, P):=\left\{X:\left.\Omega \rightarrow \mathbb{R}|E| X\right|^{2}<\infty\right\}$ (denoted by $L^{2}(P)$ from now on), which is again a Hilbert space with the norm $\|X\|_{L^{2}}:=\sqrt{E\left(|X|^{2}\right)}$. For $h \in \ell_{2}$, let $\Phi(h):=\sum_{i=1}^{\infty} h_{i} \varepsilon_{i}$, where the infinite sum in $\Phi(h)$ has to be understood as the limit in $L^{2}(P)$ of the finite sequences $\left(\sum_{i=1}^{n} h_{i} \varepsilon_{i}\right)_{n \geq 1}$. Then $\Phi$ is an isometry from $\ell_{2}$ to $L^{2}(P)$.

Assumption 2.2. We have $\|b\|_{\ell_{2}}<\infty$.

Under Assumption 2.2, we have (see (2) in [4]):

$$
E\left(\left(\sum_{i=1}^{\infty} h_{i}\left(\varepsilon_{i}-b_{i}\right)\right)^{2}\right) \leq\left(1+\|b\|_{\ell_{2}}^{2}\right)\|h\|_{\ell_{2}}^{2}<\infty,
$$

and we may consider again the infinite $\operatorname{sum}\langle h, \varepsilon-b\rangle:=\sum_{i=1}^{\infty} h_{i}\left(\varepsilon_{i}-b_{i}\right)$. Note that

$$
E(|\langle h, \varepsilon-b\rangle|) \leq \sqrt{E(\langle h, \varepsilon-b\rangle)^{2}} \leq \sqrt{1+\|b\|_{\ell_{2}}^{2}}\|h\|_{\ell_{2}} .
$$

The (self-financed) value at time 1 that can be attained starting from $x$ and using a strategy $h$ in $\ell_{2}$ with infinitely many assets is given by

$$
V^{x, h}:=x+\langle h, \varepsilon-b\rangle .
$$

Assumption 2.3. For all $i \geq 1$,

$$
P\left(\varepsilon_{i}>b_{i}\right)>0 \text { and } P\left(\varepsilon_{i}<b_{i}\right)>0 .
$$

Fix $N \geq 1$. Using Lemma 3.1 in [4, under Assumptions 2.1 and 2.3, there exists some $\alpha_{N} \in(0,1)$ such that for every $\left(h_{1}, \ldots, h_{N}\right) \in \mathbb{R}^{N}$ satisfying $\sum_{i=1}^{N} h_{i}^{2}=1$ we have

$$
P\left(\sum_{i=1}^{N} h_{i}\left(\varepsilon_{i}-b_{i}\right)<-\alpha_{N}\right)>\alpha_{N} .
$$

This condition is the so called quantitative no-arbitrage condition on any "small market" with $N$ random sources and it is well-known that this condition is equivalent to the 
existence of a equivalent martingale measure for the finite market with assets $R_{1}, \ldots, R_{N}$ (see [6] and [8]).

However, we need the existence of martingale measures for the whole market and even sufficient integrability of the martingale density. We say that EMM2 holds if

$$
\mathcal{M}_{2}:=\left\{Q \mid Q \sim P, \frac{d Q}{d P} \in L^{2}(P), E_{Q}\left(\varepsilon_{i}\right)=b_{i} \forall i \geq 1\right\} \neq \emptyset .
$$

Unfortunately, Assumptions 2.1,2.2 and 2.3 are known not to be sufficient for ensuring that EMM2 holds (see Proposition 4 of [18]). Hence we also need the following technical condition.

Assumption 2.4. We have

$$
\sup _{i \geq 1} E\left[\left|\varepsilon_{i}\right|^{3}\right]<\infty \text {. }
$$

LEMMA 2.5. Under Assumptions 2.1, 2.3 and 2.4.

$$
\text { Assumption } 2.2 \Longleftrightarrow \text { EMM2. }
$$

Proof. This is Corollary 1 of [18].

Lemma 2.6 below asserts that the quantitative no arbitrage condition, mentioned above, is true in the large market, too.

Lemma 2.6. Assume that Assumptions 2.1, 2.2, 2.3 and 2.4 hold. Then there exists some $\alpha>0$, such that for all $h \in \ell_{2}$ satisfying $\|h\|_{\ell_{2}}=1$

$$
P(\langle h, \varepsilon\rangle<-\alpha)>\alpha .
$$

Proof. This is Proposition 3.2 in [4].

REMARK 2.7. If $Q \in \mathcal{M}_{2}$ is such that $d Q / d P \in L^{2}$ and if Assumption 2.2 holds then $E_{Q}\left(V^{0, h}\right)=0$ for all $h \in \ell_{2}$, see Remark 3.1 of [4].

Lemma 2.8 below will be used in the proofs of Theorems 3.8 and 3.9 in order to show uniform integrability.

Lemma 2.8. Assume that Assumptions 2.1 and 2.2 hold and that $\sup _{i \geq 1} E\left|\varepsilon_{i}\right|^{\gamma}<\infty$ for some $\gamma \geq 2$. Then there is a constant $C_{\gamma}$ such that, for all $h \in \ell_{2}$

$$
E|\langle h, \varepsilon-b\rangle|^{\gamma} \leq C_{\gamma}\|h\|_{\ell_{2}}^{\gamma}\left(1+\|b\|_{\ell_{2}}^{\gamma}\right) .
$$

Proof. This is Lemma 3.3 in [4].

REMARK 2.9. Let $0<\lambda<\gamma$ and $c>0$. Fix $h \in \ell_{2},\|h\|_{\ell_{2}} \leq c$. Using Assumption 2.4. Hölder inequality and Lemma 2.8, we get for any $A \in \mathcal{F}$ :

$$
\begin{aligned}
E\left(\left|V^{x, h}\right|^{\lambda} 1_{A}\right) & \leq 2^{\lambda-1}|x|^{\lambda} P(A)+2^{\lambda-1} E\left(|\langle h, \varepsilon-b\rangle|^{\lambda} 1_{A}\right) \\
& \leq 2^{\lambda-1}|x|^{\lambda} P(A)+2^{\lambda-1}\left(E\left(|\langle h, \varepsilon-b\rangle|^{\gamma}\right)\right)^{\lambda / \gamma}(P(A))^{1 / q} \\
& \leq 2^{\lambda-1}|x|^{\lambda} P(A)+2^{\lambda-1} c^{\lambda}\left(C_{\gamma}\left(1+\|b\|_{\ell_{2}}^{\gamma}\right)\right)^{\lambda / \gamma}(P(A))^{1 / q},
\end{aligned}
$$

where $q$ is the conjugate of $\gamma / \lambda$. So an important consequence of Assumption 2.4 is that for any $c>0$ and $0<\lambda<3\left\{\left|V^{x, h}\right|^{\lambda} \mid h \in \ell_{2},\|h\|_{\ell_{2}} \leq c\right\}$ is uniformly integrable. 
We finally recall an important concept of functional analysis. A Banach space $\mathbb{B}$ has the Banach-Saks property if, for every norm-bounded sequence $\xi_{n} \in \mathbb{B}, n \in \mathbb{N}$, there exists a subsequence $n_{k}, k \in \mathbb{N}$, such that the corresponding arithmetic means

$$
\frac{\xi_{n_{0}}+\ldots+\xi_{n_{k-1}}}{k}
$$

converge in the norm of $\mathbb{B}$. It was proved in [1] that $L_{p}$ spaces have this property. In the present paper we will apply this result in the Hilbert space $\ell_{2}$.

3. Utility maximisation. It is standard (see [17]) to model economic agents' preferences by concave increasing utility functions $U$. So suppose that $U: \mathbb{R} \rightarrow \mathbb{R}$ is a concave strictly increasing differentiable function and that for some $x_{0} \in \mathbb{R}$

$$
U\left(x_{0}\right)=0 \text { and } U^{\prime}\left(x_{0}\right)=1 .
$$

For a claim $G \in L^{0}$ and $x \in \mathbb{R}$, we define

$$
\mathcal{A}(U, G, x):=\left\{h \in \ell_{2} \mid E U^{-}\left(V^{x, h}-G\right)<+\infty\right\} .
$$

Define the supremum of expected utility at the terminal date when delivering a contingent claim $G$, starting from initial wealth $x \in \mathbb{R}$, by

$$
u(G, x):=\sup _{h \in \mathcal{A}(U, G, x)} E U\left(V^{x, h}-G\right) .
$$

The following assumptions will be needed in Theorems 3.8 and 3.9

Assumption 3.1. There exist some constants $C_{1} \in(0, \infty), C_{2} \in \mathbb{R}_{+}$and $\beta>1$ such that for all $x \leq x_{0}$

$$
|U(x)| \geq C_{1}|x|^{\beta}-C_{2} .
$$

Assumption 3.2. There exist some constants $C_{3} \in(0, \infty), C_{4} \in \mathbb{R}_{+}$and $\gamma \geq \max (\beta, 2)$ such that for all $x \in \mathbb{R}$

$$
U^{-}(x) \leq C_{3}|x|^{\gamma}+C_{4}
$$

and

$$
\sup _{i \geq 1} E\left[\left|\varepsilon_{i}\right|^{\gamma}\right]<\infty
$$

Assumption 3.3. We have $G \geq 0$ a.s. and it satisfies $|E(U(x-G))|<+\infty$, for all $x \in \mathbb{R}$. REMARK 3.4. Assumption 3.3 is satisfied whenever $G$ is nonnegative, measurable and bounded. Define

$$
U(x):=-\frac{1}{\delta}\left[(x+1)^{-\delta}-1\right] 1_{\{x>0\}}-\frac{1}{\beta}\left[(1-x)^{\beta}-1\right] 1_{\{x \leq 0\}}
$$

for some $\beta \geq 2$ and $\delta>0$. Then $U$ is concave, strictly increasing, continuously differentiable and satisfies both Assumptions 3.1 and 3.2 whenever $\sup _{i \geq 1} E\left[\left|\varepsilon_{i}\right|^{\beta}\right]<\infty$. Note that Assumption 2.4 implies (8) when $2 \leq \beta \leq 3$.

REMARK 3.5. Let $U$ be concave, strictly increasing and differentiable, satisfying Assumptions $3.1,3.2$ and 3.3 Then (6) actually imposes no restriction on $U$. Indeed, as $U$ cannot be constant, there exists $x_{0} \in \mathbb{R}$ such that $U^{\prime}\left(x_{0}\right)>0$. Define

$$
V(x):=\frac{U(x)}{U^{\prime}\left(x_{0}\right)}-\frac{U\left(x_{0}\right)}{U^{\prime}\left(x_{0}\right)},
$$


which obviously satisfies [6]. Moreover,

$$
\begin{gathered}
|V(x)| \geq \frac{C_{1}}{U^{\prime}\left(x_{0}\right)}|x|^{\beta}-\frac{C_{2}}{U^{\prime}\left(x_{0}\right)}-\frac{\left|U\left(x_{0}\right)\right|}{U^{\prime}\left(x_{0}\right)}, \quad x \leq x_{0}, \\
V^{-}(x) \leq \frac{C_{3}}{U^{\prime}\left(x_{0}\right)}|x|^{\gamma}+\frac{C_{4}}{U^{\prime}\left(x_{0}\right)}+\frac{U^{+}\left(x_{0}\right)}{U^{\prime}\left(x_{0}\right)}, \quad x \in \mathbb{R}, \\
|E(V(x-G))| \leq \frac{|E(U(x-G))|}{U^{\prime}\left(x_{0}\right)}+\frac{\left|U\left(x_{0}\right)\right|}{U^{\prime}\left(x_{0}\right)}<\infty .
\end{gathered}
$$

So Assumptions 3.1, 3.2 and 3.3 hold for $V$. One may apply Theorems 3.8, 3.9 and Corollary 3.11 below to $V$ and then the same results can be deduced for $U$.

The following lemmata will be used in the proofs of Theorems 3.8 and 3.9

Lemma 3.6. Let Assumption 2.2 hold and assume $G \geq 0$ a.s. Then for all $y \in \mathbb{R}$ and $h \in \ell_{2}$

$$
U^{+}(y+\langle h, \varepsilon-b\rangle-G) \leq\left|x_{0}\right|+|y+\langle h, \varepsilon-b\rangle| .
$$

Proof. As $U$ is increasing, concave and differentiable, recalling (6), we get for all $y \in \mathbb{R}$,

$$
\begin{aligned}
U(y) & \leq U\left(\max \left(x_{0}, y\right)\right) \leq U\left(x_{0}\right)+\max \left(y-x_{0}, 0\right) U^{\prime}\left(x_{0}\right) \\
& \leq \max \left(y-x_{0}, 0\right) \leq\left|y-x_{0}\right| \leq|y|+\left|x_{0}\right| .
\end{aligned}
$$

If $h \in \ell_{2}$, then

$$
\begin{aligned}
& U^{+}(y+\langle h, \varepsilon-b\rangle-G) \leq U^{+}(y+\langle h, \varepsilon-b\rangle) \\
& \leq U^{+}(y+\langle h, \varepsilon-b\rangle) 1_{y+\langle h, \varepsilon-b\rangle \geq x_{0}}+U^{+}\left(x_{0}\right) 1_{y+\langle h, \varepsilon-b\rangle<x_{0}} \\
& =U(y+\langle h, \varepsilon-b\rangle) 1_{y+\langle h, \varepsilon-b\rangle \geq x_{0}} \leq\left|x_{0}\right|+|y+\langle h, \varepsilon-b\rangle| .
\end{aligned}
$$

Lemma 3.7 asserts that an optimal solution for 7 must be bounded.

Lemma 3.7. Assume that Assumptions 2.1, 2.2, 2.3, 2.4, 3.1 and 3.3 hold. Let $x \in \mathbb{R}$. There exists some constant $M_{x, G}>0$ such that if $h \in \ell_{2}$ satisfies

$$
\|h\|_{\ell_{2}}>M_{x, G}
$$

then the 0 strategy performs better than $h$, that is,

$$
E U(x-G)>E U(x+\langle h, \varepsilon-b\rangle-G) .
$$

Proof. Let $x \in \mathbb{R}$ and $h \in \ell_{2}$. Recall $\alpha>0$ from Lemma 2.6. As $b \in \ell_{2}$, there exists some $n_{\alpha} \geq 1$ such that $\left(\sum_{i \geq n_{\alpha}+1} b_{i}^{2}\right)^{1 / 2} \leq \alpha / 2$. Let

$$
\begin{aligned}
\underline{h} & :=\left(h_{1}, \ldots, h_{n_{\alpha}}, 0, \ldots\right) \quad \text { and } \quad \underline{b}=:\left(b_{1}, \ldots, b_{n_{\alpha}}, 0, \ldots\right) \\
\bar{h} & :=\left(0, \ldots, 0, h_{n_{\alpha}+1}, \ldots\right) \quad \text { and } \quad \bar{b}=:\left(0, \ldots, 0, b_{n_{\alpha}+1}, \ldots\right) .
\end{aligned}
$$

From the no-arbitrage condition in the market with $n_{\alpha}$ assets (see (2)) there exists $\alpha_{n_{\alpha}}$ such that $P(A)>\alpha_{n_{\alpha}}$, where $A:=\left\{\sum_{i=1}^{n_{\alpha}} h_{i}\left(\varepsilon_{i}-b_{i}\right)<-\alpha_{n_{\alpha}}\|\underline{h}\|_{\ell_{2}}\right\}$. Let $B:=$ $\left\{\sum_{i \geq n_{\alpha}+1} h_{i} \varepsilon_{i} \leq-\alpha\|\bar{h}\|_{\ell_{2}}\right\}$ then $P(B)>\alpha$ (recall Lemma 2.6. As the $\left(\varepsilon_{i}\right)_{i \geq 1}$ are 
independent, we see that $P(A \cap B)=P(A) P(B)>\alpha_{n_{\alpha}} \alpha$. On $A \cap B$,

$$
\begin{aligned}
\langle h, \varepsilon-b\rangle & =\langle\underline{h}, \varepsilon-b\rangle+\langle\bar{h}, \varepsilon-b\rangle \leq-\alpha_{n_{\alpha}}\|\underline{h}\|_{\ell_{2}}-\alpha\|\bar{h}\|_{\ell_{2}}-\langle\bar{h}, \bar{b}\rangle \\
& \leq-\alpha_{n_{\alpha}}\|\underline{h}\|_{\ell_{2}}-\alpha\|\bar{h}\|_{\ell_{2}}+\|\bar{b}\|_{\ell_{2}}\|\bar{h}\|_{\ell_{2}} \\
& \leq-\alpha_{n_{\alpha}}\|\underline{h}\|_{\ell_{2}}-\alpha\|\bar{h}\|_{\ell_{2}}+\alpha / 2\|\bar{h}\|_{\ell_{2}} \leq-\bar{\alpha}\left(\|\underline{h}\|_{\ell_{2}}+\|\bar{h}\|_{\ell_{2}}\right),
\end{aligned}
$$

where $\bar{\alpha}=\inf \left(\alpha_{n_{\alpha}}, \alpha / 2\right)$. Thus $P\left(\langle h, \varepsilon-b\rangle<-\bar{\alpha}\left(\|\underline{h}\|_{\ell_{2}}+\|\bar{h}\|_{\ell_{2}}\right)\right)>\alpha_{n_{\alpha}} \alpha$. Assume that $\|\underline{h}\|_{\ell_{2}}+\|\bar{h}\|_{\ell_{2}} \geq \max \left(\frac{x-x_{0}}{\bar{\alpha}}, \frac{|x|}{\bar{\alpha}}\right)$. Then applying Lemma 3.6 and Assumption 3.1 we get

$$
\begin{aligned}
E U\left(V^{x, h}-G\right) \leq E\left(U(x+\langle h, \varepsilon-b\rangle) 1_{\langle h, \varepsilon-b\rangle<-\bar{\alpha}\left(\|\underline{h}\|_{\ell_{2}}+\|\bar{h}\|_{\ell_{2}}\right)}\right) \\
\quad+E\left(U^{+}(x+\langle h, \varepsilon-b\rangle) 1_{\langle h, \varepsilon-b\rangle \geq-\bar{\alpha}\left(\|\underline{h}\| \ell_{\ell_{2}}+\|\bar{h}\|_{\ell_{2}}\right)}\right) \\
\leq U\left(x-\bar{\alpha}\left(\|\underline{h}\|_{\ell_{2}}+\|\bar{h}\|_{\ell_{2}}\right)\right) \alpha_{n_{\alpha}} \alpha+\left|x_{0}\right|+E|x+\langle\underline{h}, \varepsilon-b\rangle+\langle\bar{h}, \varepsilon-b\rangle| \\
\leq U\left(x-\bar{\alpha}\left(\|\underline{h}\|_{\ell_{2}}+\|\bar{h}\|_{\ell_{2}}\right)\right) \alpha_{n_{\alpha}} \alpha+\left|x_{0}\right|+|x|+\|\underline{h}\|_{\ell_{2}} \sqrt{1+\|\underline{b}\|_{\ell_{2}}^{2}}+\|\bar{h}\|_{\ell_{2}} \sqrt{1+\|\bar{b}\|_{\ell_{2}}^{2}} \\
\leq\left(-C_{1}\left|\bar{\alpha}\left(\|\underline{h}\|_{\ell_{2}}+\|\bar{h}\|_{\ell_{2}}\right)-x\right|^{\beta}+C_{2}\right) \alpha_{n_{\alpha}} \alpha+\left|x_{0}\right|+|x|+\left(\|\underline{h}\|_{\ell_{2}}+\|\bar{h}\|_{\ell_{2}}\right) \sqrt{1+\|b\|_{\ell_{2}}^{2}} \\
\leq\left(-C_{1} \bar{\alpha}^{\beta}\left(\|\underline{h}\|_{\ell_{2}}+\|\bar{h}\|_{\ell_{2}}\right)^{\beta}+C_{2}\right) \alpha_{n_{\alpha}} \alpha+\left|x_{0}\right|+|x|+\left(\|\underline{h}\|_{\ell_{2}}+\|\bar{h}\|_{\ell_{2}}\right) \sqrt{1+\|b\|_{\ell_{2}}^{2}},
\end{aligned}
$$

because $U\left(x-\bar{\alpha}\left(\|\underline{h}\|_{\ell_{2}}+\|\bar{h}\|_{\ell_{2}}\right)\right) \leq U\left(x_{0}\right)=0$ and

$$
\begin{aligned}
\left|\bar{\alpha}\left(\|\underline{h}\|_{\ell_{2}}+\|\bar{h}\|_{\ell_{2}}\right)-x\right|^{\beta} & \geq\left|\bar{\alpha}\left(\|\underline{h}\|_{\ell_{2}}+\|\bar{h}\|_{\ell_{2}}\right)-\right| x \|^{\beta}=\left(\bar{\alpha}\left(\|\underline{h}\|_{\ell_{2}}+\|\bar{h}\|_{\ell_{2}}\right)-|x|\right)^{\beta} \\
& \geq \bar{\alpha}^{\beta}\left(\|\underline{h}\|_{\ell_{2}}+\|\bar{h}\|_{\ell_{2}}\right)^{\beta} .
\end{aligned}
$$

Assume that

$$
\begin{gathered}
\left(\|\underline{h}\|_{\ell_{2}}+\|\bar{h}\|_{\ell_{2}}\right) \sqrt{1+\|b\|_{\ell_{2}}^{2}}-\frac{C_{1}}{2} \alpha_{n_{\alpha}} \alpha \bar{\alpha}^{\beta}\left(\|\underline{h}\|_{\ell_{2}}+\|\bar{h}\|_{\ell_{2}}\right)^{\beta}<0 \\
-\frac{C_{1}}{2} \bar{\alpha}^{\beta} \alpha_{n_{\alpha}} \alpha\left(\|\underline{h}\|_{\ell_{2}}+\|\bar{h}\|_{\ell_{2}}\right)^{\beta}+\left|x_{0}\right|+|x|+C_{2} \alpha_{n_{\alpha}} \alpha<-|E U(x-G)| \leq E U(x-G),
\end{gathered}
$$

which is true if $\|\underline{h}\|_{\ell_{2}}+\|\bar{h}\|_{\ell_{2}}>\bar{M}_{x, G}$, where

$$
\bar{M}_{x, G}:=\max \left(\left(2 \frac{\left|x_{0}\right|+|x|+C_{2} \alpha_{n_{\alpha}} \alpha+|E(U(x-G))|}{C_{1} \alpha_{n_{\alpha}} \alpha \bar{\alpha}^{\beta}}\right)^{1 / \beta},\left(2 \frac{\sqrt{1+\|b\|_{\ell_{2}}^{2}}}{C_{1} \alpha_{n_{\alpha}} \alpha \bar{\alpha}^{\beta}}\right)^{1 /(\beta-1)}\right) .
$$

Then, setting $M_{x, G}:=\max \left(\frac{x-x_{0}}{\bar{\alpha}}, \frac{|x|}{\bar{\alpha}}, \bar{M}_{x, G}\right)$, if $\|\underline{h}\|_{\ell_{2}}+\|\bar{h}\|_{\ell_{2}}>M_{x, G}$,

$$
E U\left(V^{x, h}-G\right)<E U(x-G)
$$

so the strategy 0 performs better than $h$. It follows that $\|h\|_{\ell_{2}}>M_{x, G}$ implies 10 since

$$
\|h\|_{\ell_{2}}=\left(\|\underline{h}\|_{\ell_{2}}^{2}+\|\bar{h}\|_{\ell_{2}}^{2}\right)^{1 / 2} \leq\|\underline{h}\|_{\ell_{2}}+\|\bar{h}\|_{\ell_{2}} \text {. }
$$

Now we present our first main result. We establish the existence of an optimizer for the utility maximization problem. In [19] this was shown assuming uniformly bounded exponential moments for the $\varepsilon_{i}$. In [20] the moment condition was weak but it was assumed that all the $\varepsilon_{i}$ take arbitrarily large negative and positive values. Here we do not need the latter assumption and merely assume (4) and (8). 
Theorem 3.8. Assume that Assumptions 2.1, 2.2, 2.3, 2.4, 3.1, 3.2 and 3.3 hold. Let $x \in \mathbb{R}$. There exists $h^{*} \in \mathcal{A}(U, G, x)$ such that

$$
u(G, x)=E U\left(V^{x, h^{*}}-G\right) .
$$

Proof. Let $x \in \mathbb{R}$ and let $h_{n} \in \mathcal{A}(U, G, x)$ be a sequence such that

$$
E U\left(V^{x, h_{n}}-G\right) \uparrow u(G, x), \quad n \rightarrow \infty .
$$

If $\left\|h_{n}\right\|_{\ell_{2}}>M_{x, G}$, then using Lemma 3.7. we can replace $h_{n}$ by 0 and still have a maximising sequence. So one can assume that $\sup _{n \in \mathbb{N}}\left\|h_{n}\right\|_{\ell_{2}} \leq M_{x, G}<\infty$. Hence as $\ell_{2}$ has the Banach-Saks Property, there exists a subsequence $\left(n_{k}\right)_{k \geq 0}$ and some $h^{*} \in \ell_{2}$ such that for $\widetilde{h}_{n}:=\frac{1}{n} \sum_{k=0}^{n-1} h_{n_{k}}$

$$
\left\|\widetilde{h}_{n}-h^{*}\right\|_{\ell_{2}} \rightarrow 0, \quad n \rightarrow \infty .
$$

Using (1), we get

$$
E\left\langle\widetilde{h}_{n}-h^{*}, \varepsilon-b\right\rangle^{2} \leq\left\|\widetilde{h}_{n}-h^{*}\right\|_{\ell_{2}}^{2}\left(1+\|b\|_{\ell_{2}}^{2}\right) \rightarrow 0,
$$

when $n \rightarrow \infty$. In particular, $\left\langle\widetilde{h}_{n}-h^{*}, \varepsilon-b\right\rangle \rightarrow 0, n \rightarrow \infty$ in probability. Hence also $U\left(V^{x, \widetilde{h}_{n}}-G\right) \rightarrow U\left(V^{x, h^{*}}-G\right)$ in probability by continuity of $U$. We claim that the family $U^{+}\left(V^{x, \widetilde{h}_{n}}-G\right), n \in \mathbb{N}$, is uniformly integrable. Indeed, from (9)

$$
U^{+}\left(V^{x, \widetilde{h}_{n}}-G\right) \leq\left|x_{0}\right|+\left|V^{x, \widetilde{h}_{n}}\right| .
$$

We know that $\sup _{n \in \mathbb{N}}\left\|\widetilde{h}_{n}\right\|_{\ell_{2}} \leq M_{x, G}<\infty$. Hence from Assumption 2.4 (see Lemma 2.8 and Remark 2.99, we infer that $\left\{U^{+}\left(V^{x, \widetilde{h}_{n}}-G\right), h_{n} \in \ell_{2},\left\|\widetilde{h}_{n}\right\|_{\ell_{2}} \leq M_{x, G}\right\}$ is uniformly integrable. Fatou's lemma used for $-U^{-}$implies that

$$
E\left(-U^{-}\left(V^{x, h^{*}}-G\right)\right) \geq \limsup _{n \rightarrow \infty} E\left(-U^{-}\left(V^{x, \widetilde{h}_{n}}-G\right)\right),
$$

and uniform integrability guarantees that

$$
\lim _{n \rightarrow \infty} E\left(U^{+}\left(V^{x, \widetilde{h}_{n}}-G\right)\right)=E\left(U^{+}\left(V^{x, h^{*}}-G\right)\right) .
$$

Thus, by concavity of $U$

$$
E U\left(V^{x, h^{*}}-G\right) \geq \limsup _{n \rightarrow \infty} E U\left(V^{x, \widetilde{h}_{n}}-G\right) \geq \lim _{n \rightarrow \infty} E U\left(V^{x, h_{n}}-G\right)=u(G, x),
$$

and the proof will be finished as soon as we show $h^{*} \in \mathcal{A}(U, G, x)$. From Assumption 3.2 and Lemma 2.8

$$
\begin{aligned}
E U^{-}\left(V^{x, \widetilde{h}_{n}}-G\right) & \leq C_{3} E\left|V^{x, \widetilde{h}_{n}}-G\right|^{\gamma}+C_{4} \\
& \leq C_{3}\left(2^{\gamma-1}\left(|x|^{\gamma}+E\left|<\widetilde{h}_{n}, \varepsilon-b>\right|^{\gamma}\right)\right)+C_{4} \\
& \leq C_{3}\left(2^{\gamma-1}\left(|x|^{\gamma}+C_{\gamma} M_{x, G}^{\gamma}\left(1+\|b\|_{\ell_{2}}^{\gamma}\right)\right)\right)+C_{4}=: K .
\end{aligned}
$$

Fatou's lemma used for $U^{-}$implies that

$$
E\left(U^{-}\left(V^{x, h^{*}}-G\right)\right) \leq \liminf _{n \rightarrow \infty} E\left(U^{-}\left(V^{x, \widetilde{h}_{n}}-G\right)\right) \leq K .
$$


We consider now the problem of optimization in the small market $n$ with only the random sources $\left(\varepsilon_{i}\right)_{1 \leq i \leq n}$. Let

$$
\mathcal{A}_{n}(U, G, x):=\left\{h \in \ell_{2} \mid h_{i}=0 \forall i \geq n+1, E U^{-}\left(V^{x, h}-G\right)<+\infty\right\} .
$$

Note that $\mathcal{A}_{n}(U, G, x) \subset \mathcal{A}_{n+1}(U, G, x) \subset \ldots \subset \mathcal{A}(U, G, x)$. We set for $n \in \mathbb{N}$

$$
u_{n}(G, x):=\sup _{h \in \mathcal{A}_{n}(U, G, x)} E U\left(V^{x, h}-G\right) .
$$

Now we arrive at the principal message of our paper: optimization problems in the small markets behave consistently with those on the big market, in a natural way.

TheOrem 3.9. Assume that Assumptions 2.1, 2.2, 2.3, 2.4, 3.1, 3.2 and 3.3 hold. Then for each $x \in \mathbb{R}$, we have $u_{n}(G, x) \uparrow u(G, x), n \rightarrow \infty$.

Let $h_{n}^{*}$ be an optimal solution for 1211 . Then there exists a subsequence $\left(n_{k}\right)_{k \geq 0}$ and some $\widehat{h} \in \ell_{2}$, optimal solution of (7), such that for $\widehat{h}_{n}:=\frac{1}{n} \sum_{k=0}^{n-1} h_{n_{k}}^{*}$,

$$
\left\|\widehat{h}_{n}-\hat{h}\right\|_{\ell_{2}} \rightarrow 0, n \rightarrow \infty \text {. }
$$

Proof. The sequence $u_{n}(G, x), n \in \mathbb{N}$ is clearly non-decreasing and it is bounded from above by $u(G, x)$. Let $\bar{h}_{n}:=\left(\widetilde{h}_{0}, \ldots, \widetilde{h}_{\tilde{n}}, 0, \ldots\right), n \in \mathbb{N}$ where $\widetilde{h}$ is the optimizer constructed in Theorem 3.8 Using (1) and $\widetilde{h} \in \ell_{2}$, we have

$$
E\left\langle\bar{h}_{n}-\widetilde{h}, \varepsilon-b\right\rangle^{2} \rightarrow 0, \quad n \rightarrow \infty
$$

hence also $\left\langle\bar{h}_{n}, \varepsilon-b\right\rangle \rightarrow\langle\widetilde{h}, \varepsilon-b\rangle, n \rightarrow \infty$ in probability. The Fatou lemma for $U^{+}$shows that

$$
E U^{+}\left(V^{x, \widetilde{h}}-G\right) \leq \liminf _{n \rightarrow \infty} E U^{+}\left(V^{x, \bar{h}_{n}}-G\right) .
$$

Now we show that the family $U^{-}\left(V^{x, \bar{h}_{n}}-G\right), n \in \mathbb{N}$ is uniformly integrable. Assumption 3.2 implies that

$$
\begin{aligned}
U^{-}\left(V^{x, \bar{h}_{n}}-G\right) & \leq C_{3}\left|V^{x, \bar{h}_{n}}-G\right|^{\gamma}+C_{4} \\
& \leq C_{3}\left(2^{\gamma-1}\left(|x|^{\gamma}+\left|\left\langle\bar{h}_{n}, \varepsilon-b\right\rangle\right|^{\gamma}\right)\right)+C_{4} .
\end{aligned}
$$

As $\widetilde{h}$ is optimal, $\left\|\bar{h}_{n}\right\|_{\ell_{2}} \leq\|\widetilde{h}\|_{\ell_{2}} \leq M_{x, G}$ (see Lemma 3.7) and as in Remark 2.9. $U^{-}\left(V^{x, \bar{h}_{n}}-G\right), n \in \mathbb{N}$ is uniformly integrable. We also get as in (11) that

$$
E U^{-}\left(V^{x, \bar{h}_{n}}-G\right) \leq K
$$

and $\bar{h}_{n} \in \mathcal{A}_{n}(G, U, x)$ follows. Uniform integrability implies that

$$
E U^{-}\left(V^{x, \widetilde{h}}-G\right)=\lim _{n \rightarrow \infty} E U^{-}\left(V^{x, \bar{h}_{n}}-G\right)
$$

It follows that

$$
u(G, x)=E U\left(V^{x, \widetilde{h}}-G\right) \leq \liminf _{n \rightarrow \infty} E U\left(V^{x, \bar{h}_{n}}-G\right) \leq \lim _{n \rightarrow \infty} u_{n}(G, x) \leq u(G, x) .
$$

Let $h_{n}^{*} \in \mathcal{A}_{n}(U, G, x)$ be an optimal solution for 12 . By Lemma 3.7, $\left\|h_{n}^{*}\right\|_{\ell_{2}} \leq M_{x, G}$. We proceed as in the proof of Theorem 3.8 By the Banach-Saks Property, there exists a subsequence $\left(n_{k}\right)_{k \geq 0}$ such that for $\widehat{h}_{n}:=\frac{1}{n} \sum_{k=0}^{n-1} h_{n_{k}}^{*}$,

$$
\left\|\widehat{h}_{n}-\widehat{h}\right\|_{\ell_{2}} \rightarrow 0, \quad n \rightarrow \infty,
$$

\footnotetext{
${ }^{1}$ which exists by the argument of Theorem 3.8
} 
for some $\widehat{h} \in \ell_{2}$. The arguments of the proof of Theorem 3.8 apply verbatim and show that $\widehat{h}$ is an optimizer for the utility maximization problem $(7)$ in the large market.

REMARK 3.10. When $U$ is strictly concave then the optimizer is unique and hence $h^{*}$ of Theorem 3.8 equals $\widehat{h}$ of Theorem 3.9

The corollary below addresses the problem of convergence of the reservation prices $p_{n}, p$. These latter were introduced in [9].

Corollary 3.11. Assume that Assumptions 2.1, 2.2, 2.3, 2.4, 3.1, 3.2 and 3.3 hold. The reservation price $p_{n}$ (resp. $p$ ) of $G$ in the market with the random sources $\left(\varepsilon_{i}\right)_{1 \leq i \leq n}$ (resp. with $\left.\left(\varepsilon_{i}\right)_{i \geq 1}\right)$ is defined as a solution of

$$
u_{n}\left(G, x+p_{n}\right)=u_{n}(0, x) \quad \text { and } \quad u(G, x+p)=u(0, x) .
$$

These quantities are well-defined and we have $p_{n} \rightarrow p, n \rightarrow \infty$.

Proof. We justify the definition of $p$, the case of $p_{n}$ being completely analogous. We show that the set $\{u(G, x) \mid x \in \mathbb{R}\}$ is the same as $\{u(0, x) \mid x \in \mathbb{R}\}$.

We claim that $u(G, x), u(0, x)$ are finite for all $x$. Indeed, Assumption 3.3, Lemmata 3.6 and 3.7 imply that $-\infty<u(G, x) \leq u(0, x)<\infty$. As $u$ is monotone, furthermore it is concave and thus continuous on its effective domain, it suffices to show that

$$
u(G,-\infty)=u(0,-\infty)=-\infty, \quad u(G, \infty)=u(0, \infty)=U(\infty)
$$

and that $u(G, x), u(0, x)<U(\infty)$ for all $x$ because in this case $\{u(G, x) \mid x \in \mathbb{R}\}=$ $\{u(0, x) \mid x \in \mathbb{R}\}=(-\infty, U(\infty))$.

We first concentrate on the latter claim. If $U(\infty)=\infty$ then this is obvious. Otherwise denote by $h^{\prime}, h^{\prime \prime}$ the strategies attaining $u(0, x), u(G, x)$, respectively. Then, by the strictly increasing property of $U$, we have

$$
u(0, x)=E U\left(x+\left\langle h^{\prime}, \varepsilon-b\right\rangle\right)<E U(\infty)=U(\infty)
$$

and

$$
u(G, x)=E U\left(x+\left\langle h^{\prime \prime}, \varepsilon-b\right\rangle-G\right)<E U(\infty)=U(\infty) .
$$

Now we turn to showing (13). It is clear that $u(G, \infty), u(0, \infty) \leq U(\infty)$ and

$$
u(0, \infty)=\lim _{x \rightarrow \infty} u(0, x) \geq \lim _{x \rightarrow \infty} U(x)=U(\infty) .
$$

Assumption 3.3 and Fatou's lemma also imply that

$$
u(G, \infty) \geq \liminf _{x \rightarrow \infty} u(G, x) \geq \liminf _{x \rightarrow \infty} E U(x-G) \geq U(\infty) .
$$

Since $u(G, x) \leq u(0, x)$, it is enough to establish $\lim _{x \rightarrow-\infty} u(0, x)=-\infty$. By concavity, this is clearly the case if $u(0, \cdot)$ is not the constant function. But if $u(0, \cdot)=c$ then we would necessarily have $c \geq U(\infty)$ by 15 which contradicts (14).

We now turn to proving convergence. Arguing by contradiction let us assume that, along a subsequence (which we continue to denote by $n$ ), one has $p_{n} \rightarrow p$ for some $p<p$ (the case of a limit $\bar{p}>p$ is analogous). It follows that there is $N$ such that, for $n \geq N$, $p_{n}<(p+\underline{p}) / 2<p$. Using Theorem 3.8. let $h^{\dagger} \in \mathcal{A}(G, U, x+(p+\underline{p}) / 2) \subset \mathcal{A}(G, U, x+p)$ satisfy

$$
u(G, x+(p+\underline{p}) / 2)=E U\left(x+(p+\underline{p}) / 2+\left\langle h^{\dagger}, \varepsilon-b\right\rangle-G\right) .
$$


Then, the definition of the reservation prices and Theorem 3.9 imply that

$$
\begin{aligned}
& \limsup _{n \rightarrow \infty} u_{n}\left(G, x+p_{n}\right) \leq \limsup _{n \rightarrow \infty} u_{n}(G, x+(p+\underline{p}) / 2) \\
& =u(G, x+(p+\underline{p}) / 2)=E U\left(x+(p+\underline{p}) / 2+\left\langle h^{\dagger}, \varepsilon-b\right\rangle-G\right) \\
& <E U\left(x+p+\left\langle h^{\dagger}, \varepsilon-b\right\rangle-G\right) \leq u(G, x+p) \\
& =u(0, x)=\lim _{n \rightarrow \infty} u_{n}(0, x)=\lim _{n \rightarrow \infty} u_{n}\left(G, x+p_{n}\right),
\end{aligned}
$$

a gross contradiction.

Acknowledgments. M.R. was supported by the National Research, Development and Innovation Office, Hungary [Grant KH 126505] and by the "Lendület" programme of the Hungarian Academy of Sciences [Grant LP 2015-6].

\section{References}

[1] S. Banach, S. Saks, Sur la convergence forte dans les champs $L^{p}$, Studia Math. 2 (1930), $51-57$.

[2] M. Baran, Asymptotic pricing in large financial markets, Math. Methods Oper. Res. 66 (2007), 1-20.

[3] L. Campi, Mean-variance hedging in large financial markets, Stoch. Anal. Appl. 27 (2009), 1129-1147.

[4] L. Carassus, M. Rásonyi, Risk-neutral pricing for arbitrage pricing theory, J. Optim. Theory Appl. 186 (2020), 248-263.

[5] C. Cuchiero, I. Klein, J. Teichmann, A new perspective on the fundamental theorem of asset pricing for large financial markets, Theory Probab. Appl. 60 (2016), 561-579.

[6] R. C. Dalang, A. Morton, W. Willinger, Equivalent martingale measures and no-arbitrage in stochastic securities market models, Stochastics Stochastics Rep. 29 (1990), 185-201.

[7] M. De Donno, P. Guasoni, M. Pratelli, Superreplication and utility maximization in large financial markets, Stochastic Process. Appl. 115 (2005), 2006-2022.

[8] H. Föllmer, A. Schied, Stochastic Finance: an Introduction in Discrete Time, Walter de Gruyter, Berlin, 2002.

[9] R. Hodges, K. Neuberger, Optimal replication of contingent claims under transaction costs, Rev. Futures Mkts. 8 (1989), 222-239.

[10] Yu. M. Kabanov, D. O. Kramkov, Large financial markets: asymptotic arbitrage and contiguity, Teor. Veroyatnost. i Primenen. 39 (1994), 222-229; English transl.: Theory Probab. Appl. 39 (1994), 182-187.

[11] Yu. M. Kabanov, D. O. Kramkov, Asymptotic arbitrage in large financial markets, Finance Stoch. 2 (1998), 143-172.

[12] I. Klein, A fundamental theorem of asset pricing for large financial markets, Math. Finance 10 (2000), 443-458.

[13] I. Klein, W. Schachermayer, Asymptotic arbitrage in non-complete large financial markets, Teor. Veroyatnost. i Primenen. 41 (1996), 927-934; reprinted in: Theory Probab. Appl. 41 (1996), 780-788.

[14] I. Klein, W. Schachermayer, A quantitative and a dual version of the Halmos-Savage theorem with applications to mathematical finance, Ann. Probab. 24 (1996), 867-881. 
[15] J. Lintner, The valuation of risky assets and the selection of risky investments in stock portfolios and capital budgets, Rev. Econom. Statist. 47 (1965), 13-37.

[16] O. Mostovyi, Utility maximization in a large market, Math. Finance 28 (2018), 106-118.

[17] J. von Neumann, O. Morgenstern, Theory of Games and Economic Behavior, Princeton Univ. Press, Princeton, NJ, 1944.

[18] M. Rásonyi, Arbitrage pricing theory and risk-neutral measures, Decis. Econ. Finance 27 (2004), 109-123.

[19] M. Rásonyi, On optimal strategies for utility maximizers in the Arbitrage Pricing Model, Int. J. Theor. Appl. Finan. 19 (2016), 1650047.

[20] M. Rásonyi, Maximizing expected utility in the Arbitrage Pricing Model, J. Math. Anal. Appl. 454 (2017), 127-143.

[21] S. A. Ross, The arbitrage theory of capital asset pricing, J. Econom. Theory 13 (1976), 341-360.

[22] W. Sharpe, Capital asset prices: a theory of market equilibrium under conditions of risk, J. Finance 19 (1964), 425-442. 